\title{
Real-Space Observation of Ultraslow Light in Photonic Crystal Waveguides
}

\author{
H. Gersen, ${ }^{1, *}$ T. J. Karle, ${ }^{2}$ R. J. P. Engelen, ${ }^{1}$ W. Bogaerts, ${ }^{3}$ J. P. Korterik, ${ }^{1}$ N. F. van Hulst, ${ }^{1}$ T. F. Krauss, ${ }^{2}$ and L. Kuipers ${ }^{1,4, \dagger}$ \\ ${ }^{1}$ Applied Optics Group, Department of Science Technology and MESA ${ }^{+}$Institute for Nanotechnology, University of Twente, \\ P.O. Box 217, 7500 AE Enschede, The Netherlands \\ ${ }^{2}$ Ultrafast Photonics Collaboration, School of Physics and Astronomy, University of St. Andrews, St. Andrews, \\ Fife, KY16 9SS, United Kingdom \\ ${ }^{3}$ Ghent University-IMEC, Department of Information Technology (INTEC), B-9000 Gent, Belgium \\ ${ }^{4}$ FOM-Institute for Atomic and Molecular Physics (AMOLF), Kruislaan 407, 1098 SJ Amsterdam, The Netherlands \\ (Received 3 September 2004; published 25 February 2005)
}

\begin{abstract}
We show the real-space observation of fast and slow pulses propagating inside a photonic crystal waveguide by time-resolved near-field scanning optical microscopy. Local phase and group velocities of modes are measured. For a specific optical frequency we observe a localized pattern associated with a flat band in the dispersion diagram. During at least 3 ps, movement of this field is hardly discernible: its group velocity would be at most $c / 1000$. The huge trapping times without the use of a cavity reveal new perspectives for dispersion and time control within photonic crystals.
\end{abstract}

DOI: 10.1103/PhysRevLett.94.073903

Scientists have long been trying to capture and manipulate light with an all-optical analogue to the electronic silicon chip. Photonic band gap materials, periodic dielectric structures that mimic the properties of semiconductors to guide and trap light [1], have already enabled the creation of micrometer sized lasers [2], waveguides [3], and nanocavities [4] by confining the light on a wavelength scale. Fast signals in optical structures have a broad spectrum and therefore sample the (material) dispersion over a wide range. Photonic crystal $(\mathrm{PhC})$ structures that allow the control and compensation of dispersion therefore become increasingly important $[5,6]$. PhCs exhibiting dispersion control enable the selective retardation of optical signals via ultraslow light propagation, e.g., for optical delay lines [7], enhanced light-matter interaction [5], or quantum information processing [8].

Experimental verification of dispersive phenomena in $\mathrm{PhCs}$, however, is still a difficult and demanding issue [9]. Only indirect measurements of the group velocity and its dispersion have been reported so far [5,10-12]. In general, these methods rely either on using a reference structure or on so-called "cutback methods." As signals are collected outside the structure of interest, little direct insight into the behavior and the evolution of pulses inside the structure is obtained. To take a direct look inside a PhC is, however, far from trivial as conventional microscopy is limited by the diffraction limit. Furthermore, guided $\mathrm{PhC}$ waveguide $(\mathrm{PhCW})$ modes are evanescent in air and can therefore not be observed with far-field techniques. Circumventing these restrictions is possible by bringing a subwavelengthsized probe into the evanescent fields close to a sample surface. The few near-field scanning optical microscopy (NSOM) experiments on PhCs so far detected only field intensities [13-15]. However, letting the collected light interfere with a frequency shifted reference beam gives access to the local field amplitude and phase [16]. The
PACS numbers: 42.70.Qs, 68.37.Uv, 78.47.+p, 78.67.-n

combination with the use of ultrashort pulses (see Fig. 1) allows the direct visualization of dynamic effects inside the structure $[17,18]$.

Here, we use this technique to visualize fast and slow pulses as they propagate inside a PhCW. For a specific optical frequency, a complex, localized stationary modal pattern is observed associated with a flat band in the measured dispersion diagram. This pattern persists for more than $3.6 \mathrm{ps}$ after the excitation pulse has moved away. This constitutes a "trapping time" of the same order of magnitude as that of state-of-the-art photonic crystal cavities [4]. However, this pattern is not associated with any cavity or the like. Nevertheless, we observe that it
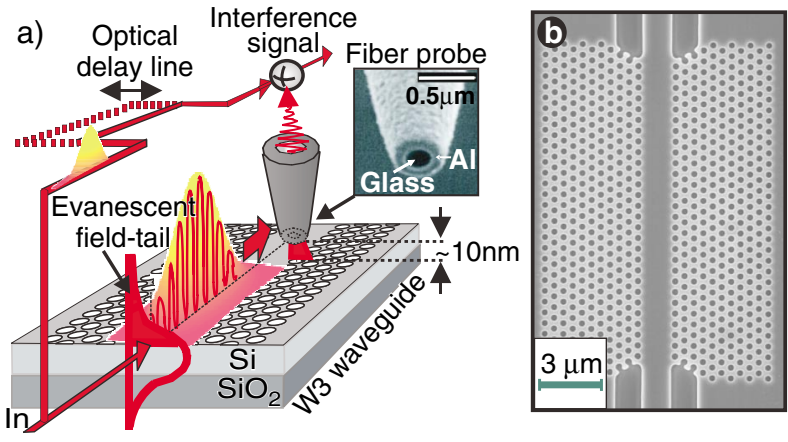

FIG. 1 (color). (a) Schematic representation of a pulse tracking experiment on a W3 $\mathrm{PhCW}$. The evanescent field of a propagating pulse is picked up by a metal coated fiber probe with a subwavelength-sized aperture and interferometrically mixed with light from a reference branch. The inset shows the probe used in the experiment. The aperture size $(\phi=240 \mathrm{~nm})$ in the first approximation determines the optical resolution. Measurements are done by raster scanning the optical probe across the structure at a constant height $(<10 \mathrm{~nm})$. (b) Top view of the $\mathrm{PhCW}$ under study, although with a shorter device length. 
moves by less than $0.9 \mu \mathrm{m}$ in $3 \mathrm{ps}$, suggesting an upper limit for the group velocity of less than $c / 1000$.

The PhCWs were fabricated on a silicon-on-insulator wafer, with a $220 \mathrm{~nm}$ thick silicon (Si) waveguide layer on top of a $1 \mu \mathrm{m}$ thick silicon dioxide $\left(\mathrm{SiO}_{2}\right)$ cladding [19]. Holes are arranged in a hexagonal array [period $a=400$ and $460 \mathrm{~nm}$, hole radius $r=130 \mathrm{~nm}$, and three rows of missing holes define the so-called W3 PhCW; see Fig. 1(b)]. Linearly TE-polarized light ( $E \|$ to crystal plane) is coupled to the $\mathrm{PhCW}$.

Figure 2 shows how ultrafast pulses can be tracked through a W3 PhCW with a local time-resolved heterodyne interference measurement. Short transform-limited Gaussian pulses (FWHM $120 \pm 10 \mathrm{fs}, \Delta \omega \approx 0.02$ ) were launched in the access waveguide at a wavelength $(\lambda)$ of $1245 \pm$ $0.5 \mathrm{~nm}(\omega=0.3695)$. In the measured topography of the $\mathrm{PhCW}$ [Fig. 2(a)] the entrance and exit waveguides are clearly resolved. One can also recognize the pattern of holes $(a=460 \mathrm{~nm})$ that forms the PhC. The measured optical amplitudes for increasing reference times are shown [Figs. 2(b)-2(g)]. We observe the separation of the incoming pulse in multiple pulses with different modal distributions. It is immediately clear that the different modes propagate with largely different group velocities [20].

The time evolution of individual modes can be extracted from these local measurements [21]. For each pulse mea-
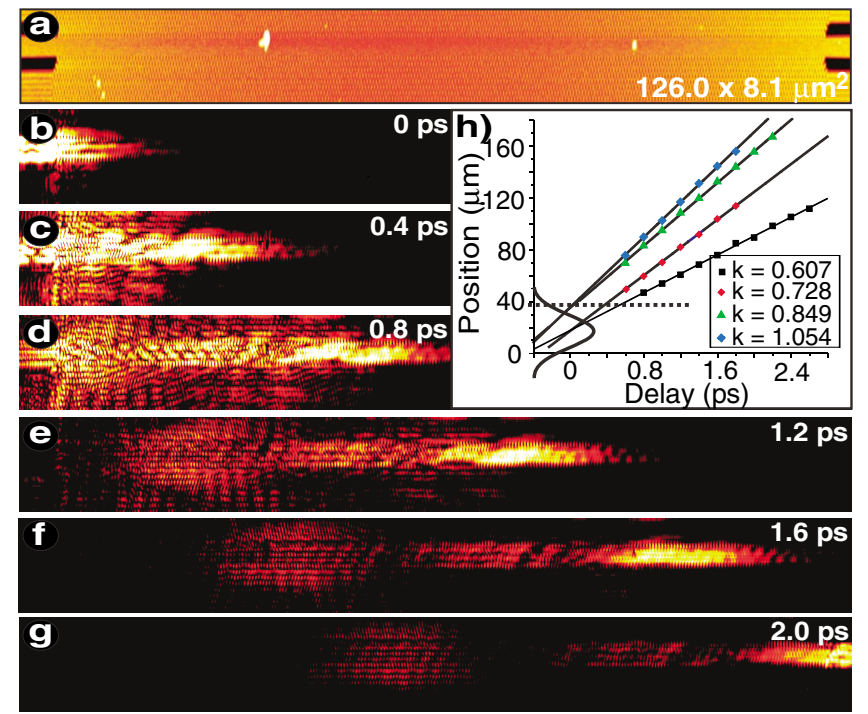

FIG. 2 (color). A time-resolved NSOM measurement on a W3 PhCW $(a=460 \mathrm{~nm})$. (a) Topographic image of the structure rotated $90^{\circ}$ with respect to Fig. 1(b). (b) - (g) The optical amplitude in the $\mathrm{W} 3 \mathrm{PhCW}$ for different reference times $(400 \pm 1 \mathrm{fs}$ between frames; all frames have the same color scale). A movie displaying the pulse propagation is available [20]. It is apparent that pulses with different modal distributions and group velocities are excited in the W3 waveguide. (h) The measured position of the center of mass for pulses with different modal distributions (and different wave vectors) as a function of the delay time. The dotted line represents the start of the $\mathrm{PhC}$. surement the raw data correspond to the amplitude times the cosine of the phase [18]. A spatial Fourier transform (SFT) directly reveals the spatial frequencies present in the raw data. The various modes excited in the waveguide show up as individual peaks in the SFT. Applying an inverse Fourier transform to a selected peak allows the spatial position of the pulse in that mode to be determined. Repeating this procedure for successive frames yields the pulse position as a function of time. When the observed pulses are roughly Gaussian and retain their shape during propagation, the change in the position of the "center of mass" (c.m.) is an unambiguous measure for the group velocity [18]. Figure 2(h) shows the position of the c.m. as a function of the reference time for several modes. A linear fit yields the group velocity of these modes. We observe a group velocity as small as $(0.121 \pm 0.001) \times c$, with $c$ the vacuum speed of light. The highest measured group velocity is $(0.243 \pm 0.001) \times c$. However, not all of the experimentally observed modes in the $\mathrm{PhCW}$ have been depicted in Fig. 2(h). For those modes ( $k=0.797,0.936$, and 0.984) the pulse shape was found to change strongly as a function of time. This is the result of a very strong group-velocity dispersion (GVD). Using an analytical model [18] we calculate that for the strongest dispersed mode at $k=$ 0.797 the GVD is at least as large as $3 \times 10^{5} \mathrm{ps}^{2} / \mathrm{km}$. The observed GVD for this specific mode is almost 5 orders of magnitude larger than in a conventional single-mode optical fiber (typically $8 \mathrm{ps}^{2} / \mathrm{km}$ at $1245 \mathrm{~nm}$ ).

Note that extrapolation of the fitted lines [Fig. 2(h)] indicates that the various modes are not excited at the same time at the start of the crystal. The observed time delay between excitation of the different modes in Fig. 2(h) can be as large as $500 \mathrm{fs}$. The time lags are caused by multiple modes in the entrance waveguide. In a conventional "time-of-flight" measurement, in which the sample is treated as a "black box," such an (in principle undesired) excitation by higher order modes could, for short device lengths, lead to the erroneous conclusion that very small group velocities are observed.

The phase sensitivity of the technique can be exploited to determine the local photonic band structure experimentally. For a certain $\omega$ a SFT of the raw data reveals the spatial frequencies and hence propagation constants. Varying $\omega$ therefore yields the band structure. Figure 3(a) displays the SFT of the data for all wavelengths accessible in our experiment (1180-1350 nm, wavelength step $5 \mathrm{~nm}$ ) on two different PhCWs $(a=400$ and $460 \mathrm{~nm}$, respectively). The vertical axis displays the excitation wavelength in terms of $\omega$, while the horizontal axis corresponds to the observed spatial frequencies. For a wide defect waveguide such as the W3, the principle components of the field follow roughly the same dispersion as a nonperiodic waveguide of identical proportions. The six positive slopes visible in Fig. 3(a) for $0.5<k<1$ agree well with the first six TE-polarized modes calculated for such a nonperiodic waveguide [22]. 


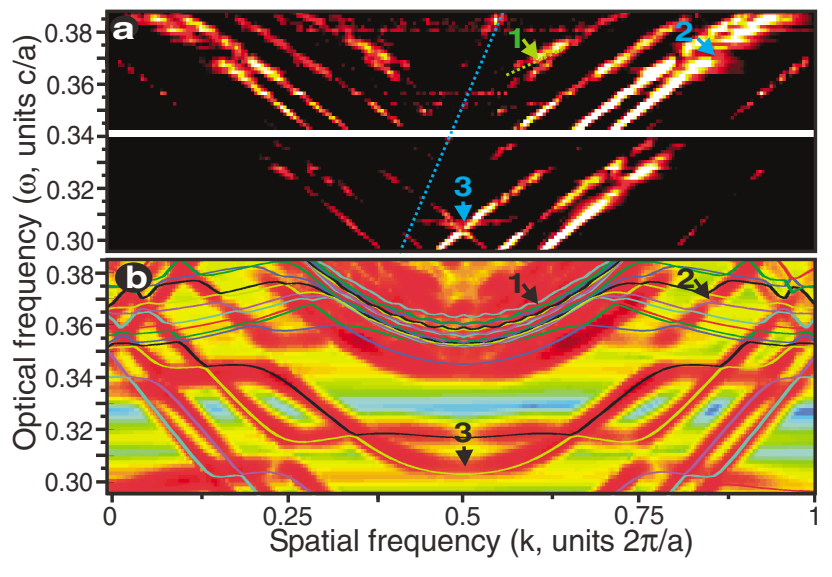

FIG. 3 (color). (a) Experimental dispersion diagram of the modes excited in the W3 PhCW. Displayed is the spatial Fourier transform of data for multiple excitation frequencies. The location of the slowest mode $(k=0.607)$ observed in Fig. 2 is indicated by 1 . Its measured group velocity gives an independent measure for the slope of its dispersion curve (green dotted line). (b) Simulated dispersion diagram. The local slope of the calculated band structure for $k=0.607$ (indicated by 1) gives an expected group velocity of $0.115 \times c$ in good agreement with the measured value of $(0.121 \pm 0.001) \times c$. Comparing Fig. 3(b) with the measured band structure in Fig. 3(a) reveals a flat band around $\omega=0.305$ (indicated by 3 ).

The effect of the periodic modulation of the refractive index on the propagating modes can be calculated by directly solving time-dependent Maxwell equations with a 2D finite-difference time domain (FDTD) method. Fourier transformation of the calculated fields for multiple excitation frequencies yields the expected band structure shown in Fig. 3(b) [23]. The overlaid solid lines depict the band structure calculated by a plane wave method. First, the periodicity introduces additional wave vector components, which are visible in Fig. 3(a) for $0<k<0.5$. Second, the folding of modes causes modes to cross, creating gaps in the lines due to the avoided crossings, for which an indication is, e.g., observed in the region indicated by 2 . Good agreement is found between the measured group velocities and the slopes $(d \omega / d k)$ in the experimental and calculated band diagrams. However, comparison of the region indicated by 3 in Fig. 3 shows that there are clear differences between experiment and theory. Preliminary calculations for a nonperiodic waveguide indicate that the positive slope visible in Fig. 3(a) at this region [and not in Fig. 3(b)] agrees with a fourth order TE mode [22].

Close inspection of the region indicated by 3 in Fig. 3(a) shows the presence of a flat $\mathrm{PhCW}$ mode at the Brillouin zone boundary. This flat band is also clearly visible in the simulated band structure depicted in Fig. 3(b). Figure 4 presents pulse tracking measurements for this flat band $(\omega=0.305)$, with 4(a) the measured topography. In Figs. 4(b)-4(k) the optical amplitude is depicted as a

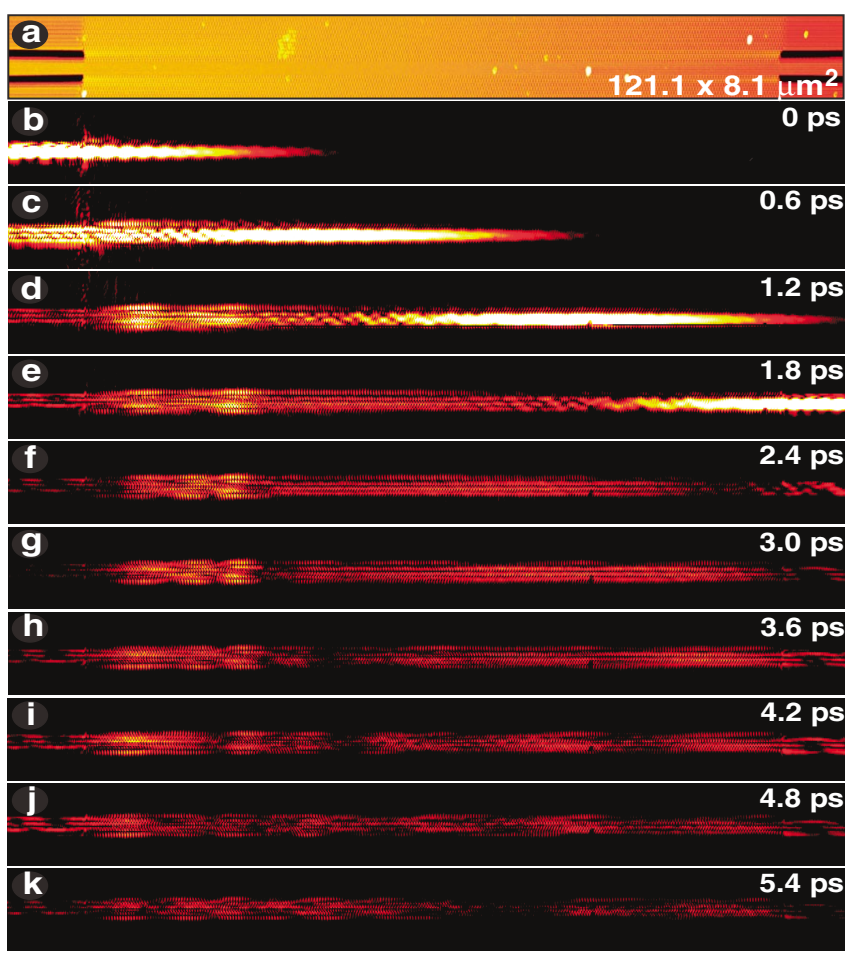

FIG. 4 (color). Pulse tracking experiment at a flat band at $\omega=$ $0.305(\lambda=1310 \mathrm{~nm}, a=400 \mathrm{~nm})$. (a) Topography of the $\mathrm{PhCW}$. (b)-(k) The optical amplitude as a function of reference time (all frames have the same color scale). A movie displaying the pulse propagation is available [20]. We observe a complex and stationary localized modal pattern in the first $25 \mu \mathrm{m}$ of the PhCW which exists for more than 3.6 ps after the excitation pulse has moved away. The field pattern moves by less than $0.9 \mu \mathrm{m}$ in $3 \mathrm{ps}$, suggesting a group velocity of less than $c / 1000$. Note that all light observed in (f) through (k) belongs to the same mode located between $k=0.4-0.6$, unlike in Fig. 2 where various discrete wave vectors are present simultaneously.

function of the reference time in steps of $600 \pm 1 \mathrm{fs}$. Several dynamic phenomena are apparent. First, we observe a first order mode pulse propagating through the structure in Figs. 4(b)-4(e). Second, in the wake of this pulse, striking effects are visible. Fascinatingly, a localized and stationary optical field is observed which persists for very long times. It is located in the first $25 \mu \mathrm{m}$ behind the entrance of the $\mathrm{PhCW}$. The pattern is reminiscent of the patterns associated with mini-stopgaps [24,25]. Despite the fact that the $\mathrm{PhCW}$ is an open structure and no resonant cavity is present, the optical field persists for more than $3.6 \mathrm{ps}$. This trapping time can be compared to the quality factor $Q$ of a resonator via $Q=\omega \tau$, where $\omega$ is the optical frequency and $\tau$ the trapping time of light. The trapping time of $3.6 \mathrm{ps}$ is equivalent to a $Q$ of $6 \times 10^{3}$. After roughly $3.6 \mathrm{ps}$ the spatial shape of the field alters, but even after 5 ps light with a comparable symmetry is still present in the structure. Between 1.2 and $4.2 \mathrm{ps}$, movement of this localized light field is hardly discernible: if a group velocity should be assigned, it would be at most $c / 1000$. 
Note that the localized field is not excited directly at the start of the $\mathrm{PhCW}$. A pulse excited directly at the start, propagating at $c / 1000$, would have taken many nanoseconds before arriving at the location where we observe it. This is clearly not the case, indicating that this is not just a simple slow pulse propagating near a photonic band edge.

Our observation clearly demonstrates that a guided mode with an extremely slow velocity and very large group-velocity dispersion is excited in the $\mathrm{PhCW}$. It is often thought that it is hard to excite such a slow mode due to the large mismatch in group velocities. Apparently, the special situation of an index guided mode with the proper modal symmetry interacting with a slow mode at the Brillouin boundary allows efficient excitation of the ultraslow light field. Although not easy, specific modes for excitation can be generated by careful design of mode converters and couplers and could lead to very efficient coupling to slow modes [26]. The huge trapping times observed without the use of a cavity should therefore open perspectives for new schemes of dispersion and time control of optical signals within photonic crystals.

This research is part of the strategic Research Orientation on Advanced Photonic Structures of the $\mathrm{MESA}^{+}$Institute for Nanotechnology. Furthermore, this work is part of the research program of the Stichting voor Fundamenteel Onderzoek der Materie (FOM), which is financially supported by the Nederlandse Organisatie voor Wetenschappelijk Onderzoek (NWO). W. B. is supported by the European Union as part of the IST-PICCO project. T.K. acknowledges support from Agilent Technologies.

*Current address: Interdisciplinary Nanoscience Center (iNANO), Center for Atomic-scale Materials Physics (CAMP) and Department of Physics and Astronomy, University of Aarhus, DK 8000 Aarhus C, Denmark.

Corresponding author.

Electronic address: 1.kuipers@amolf.nl
[1] J. D. Joannopoulos, R. D. Meade, and J. N. Winn, Photonic Crystals: Molding the Flow of Light (Princeton University Press, Princeton, NJ, 1995).

[2] O. Painter et al., Science 284, 1819 (1999).

[3] T.F. Krauss, R.M. De La Rue, and S. Brand, Nature (London) 383, 699 (1996).

[4] Y. Akahane et al., Nature (London) 425, 944 (2003).

[5] M. Notomi et al., Phys. Rev. Lett. 87, 253902 (2001).

[6] T. J. Karle et al., J. Lightwave Technol. 22, 514 (2004).

[7] M.F. Yanik and S. Fan, Phys. Rev. Lett. 92, 083901 (2004).

[8] M. D. Lukin and A. Imamoglu, Nature (London) 413, 273 (2001).

[9] X. Letartre et al., Appl. Phys. Lett. 79, 2312 (2001).

[10] A. Imhof et al., Phys. Rev. Lett. 83, 2942 (1999).

[11] Yu. A. Vlasov et al., Phys. Rev. E 60, 1030 (1999).

[12] K. Inoue et al., Phys. Rev. B 65, 121308(R) (2002).

[13] P. L. Phillips et al., J. Appl. Phys. 85, 6337 (1999).

[14] S. I. Bozhevolnyi et al., Phys. Rev. B 66, 235204 (2002).

[15] P. Kramper et al., Phys. Rev. Lett. 92, 113903 (2004).

[16] E. Flück et al., J. Lightwave Technol. 21, 1384 (2003).

[17] M. L. M. Balistreri et al., Science 294, 1080 (2001).

[18] H. Gersen et al., Phys. Rev. E 68, 026604 (2003).

[19] W. Bogaerts et al., Opt. Express 12, 1583 (2004).

[20] See EPAPS Document No. E-PRLTAO-94-078510 for a high resolution version of Fig. 2 and movies of the pulse propagation through the $\mathrm{PhCW}$. The available movies correspond to the data in Figs. 2 and 4, respectively. A direct link to this document may be found in the online article's HTML reference section. The document may also be reached via the EPAPS homepage (http://www.aip.org/ pubservs/epaps.html) or from ftp.aip.org in the directory/ epaps/. See the EPAPS homepage for more information.

[21] H. Gersen et al. Phys. Rev. E 70, 066609 (2004).

[22] Calculated by a 1D mode solver (CAMFR); width $=2 \sqrt{3} a-2 r=1.29 \mu \mathrm{m}, a=460 \mathrm{~nm}, n_{\mathrm{eff}}=2.89$.

[23] A preliminary 3D FDTD simulation including the $\mathrm{SiO}_{2}$ cladding layer yields similar results.

[24] S. Olivier et al., Phys. Rev. B 63, 113311 (2001).

[25] M. Agio and C. M. Soukoulis, Phys. Rev. E 64, 055603(R) (2001).

[26] J. Witzens and A. Scherer, J. Opt. Soc. Am. A 20, 935 (2003). 